\title{
Os efeitos dos Exercícios de Kegel em idosas com Incontinência Urinária: uma Revisão Sistemática
}

\author{
The Effects of Kegel Examinations on the Elderly with Urinary Incontinence: A Systematic \\ Review
}
Los Efectos de los Ejercicios de Kegel en ancianos con incontinencia urinaria: una revisión sistemática

\begin{abstract}
Celiane da Mota Silveira ${ }^{1 *}$, Albério Ambrósio Cavalcante ${ }^{2}$, Edna Graziele de Conceição Ribeiro².
\end{abstract}
\section{RESUMO}

Objetivo: Analisar os efeitos dos Exercícios de Kegel (EK) em idosas com Incontinência Urinária (IU) realizado pelo fisioterapeuta. Métodos: Revisão sistemática, nela realizou-se pesquisas em várias bases de dados, como: Lilacs, Medline e Scielo. Dessa forma, foram encontrados uma amostra de 11 artigos que estavam de acordo com os critérios de inclusão. Vale ressaltar que foram selecionados artigos publicados em inglês, português e espanhol no período entre 2010 e 2019. Resultados: Após a captação dos artigos foi realizada uma análise dos resultados desses estudos tendo como resultado a confirmação da satisfação das pacientes em perceber que houve uma diminuição dos seus sintomas com um tempo pequeno através do tratamento utilizando EK. Pode-se perceber também que os trabalhos no Brasil realizados sobre esse assunto ainda se encontram limitados e de amostras reduzidas. Conclusão: Assim, verifica-se a necessidade de programas que possibilite a difusão e implementação do tratamento da IU pelo fisioterapeuta, para que quando implementados possam oferecer um tratamento convencional menos oneroso, sem custo e com principal foco: a atenção à saúde da mulher, para garantir sua melhor qualidade de vida.

Palavras-chave: Incontinência Urinária, Exercícios de Kegel, Idosas, Fisioterapia.

\begin{abstract}
Objective: To analyze the effects of Kegel (EK) exercises on elderly women with urinary incontinence (UI) performed by the physiotherapist. Methods: Systematic review was carried out in several databases such as Lilacs, Medline and Scielo. Thus, a sample of 11 articles that met the inclusion criteria were found. It is noteworthy that articles published in English, Portuguese and Spanish were selected in the period between 2010 and 2019. Results: After the abstraction of the articles an analysis of the results of these studies was carried out, confirming the patients' satisfaction in perceiving that there was a decrease of their symptoms with a short time through treatment using EK. It can also be noticed that the work in Brazil carried out on this subject is still limited and of reduced samples. Conclusion: Therefore, there is a need for programs that allow the dissemination and implementation of UI treatment by the physiotherapist, so that when implemented they can offer a conventional treatment less costly, with no cost and with the main focus: women's health care, to ensure their best quality of life.
\end{abstract}

Key words: Urinary incontinence, Kegel exercises, Elderly, Physical therapy.

${ }^{1}$ Faculdade de Medicina de Juazeiro do Norte (FMJ). Juazeiro do Norte Ceará. *E-mail: celianemotta@gmail.com

SUBMETIDO EM: 4/2019

ACEITO EM: 5/2019

PUBLICADO EM: 7/2019

REAS/EJCH | Vol. Sup. 26 | e734 | DOI: https://doi.org/10.25248/reas.e734.2019 Página 1 de 8 


\section{RESUMEN}

Objetivo: Analizar los efectos de los Ejercicios de Kegel (EK) en ancianos con Incontinencia Urinaria (IU) realizado por el fisioterapeuta. Métodos: Revisión sistemática, en él se realizaron investigaciones en varias bases de datos, como: Lilacs, Medline y Scielo. De esta forma, se encontraron una muestra de 11 artículos que estaban de acuerdo con los criterios de inclusión. Es de destacar que fueron seleccionados publicados en Inglés, portugués y español en el periodo comprendido entre 2010 y 2019. Resultados: Después de la captación de los artículos se realizó un análisis de los resultados de estos estudios dando como resultado la confirmación de la satisfacción de las pacientes en percibir que hubo una disminución de sus síntomas con un tiempo pequeño a través del tratamiento utilizando EK. Se puede percibir también que los trabajos en Brasil realizados sobre ese asunto todavía se encuentran limitados y de muestras reducidas. Conclusión: Así, se verifica la necesidad de programas que posibilite la difusión e implementación del tratamiento de la IU por el fisioterapeuta, para que cuando implementados puedan ofrecer un tratamiento convencional menos oneroso, sin costo y con principal foco: la atención a la salud de la mujer, para garantizar su mejor calidad de vida.

Palabras clave: Incontinencia Urinaria, Ejercicios de Kegel, Ancianos, Fisioterapia.

\section{INTRODUÇÃO}

É evidente o aumento da população idosa na sociedade contemporânea, decorrente das mudanças ocorridas nos níveis de mortalidade e fecundidade. Dessa forma, a população passou por um regime demográfico com baixa mortalidade e, também, com baixa fecundidade, levando a um envelhecimento da população nunca visto anteriormente. Toda essa mudança mostra desafios para a saúde do país, pois surge uma maior demanda no atendimento para controlar patologias e manter a qualidade de vida do idoso (REIS LA e TORRES GV, 2011; ROIG JJ, et al. 2013).

O assoalho pélvico possui músculos que são classificados como músculos estriados esqueléticos, possuindo contração voluntária. Esses músculos exerce uma função contra a força gravitacional e a pressão intra-abdominal. Além disso, permite o suporte das vísceras pélvicas e do abdômen auxiliando de forma satisfatória na continência urinária e fecal. É valido ressaltar que uma diminuição da força dessa musculatura causa vários problemas, como por exemplo, a incontinência urinária. (ALPERIN M, et al., 2014; BRUNO GS, et al., 2016).

A Incontinência Urinária é classificada pela Sociedade Internacional de Incontinência (ICS) como Incontinência Urinária de Esforço (IUE), Incontinência Urinária de Urgência (IUU), Incontinência Urinária Mista (IUM), Incontinência Funcional, Incontinência Postural, Incontinência Insensível e Incontinência Coital. As três primeiras são as que mais ocorrem, sendo que a IUE é a forma mais comum $(50-70 \%$ dos casos) caracterizada por ocorrer após exercício físico, espirro ou tosse; a IUU se apresenta por forte desejo de urinar; e a IUM quando é seguido por apresentar a incontinência de urgência e de esforço (RODRIGUES MP, et al., 2016; TOPUZ S e SEVIG EU, 2016).

Muitas intervenções cirúrgicas e medicamentosas vêm sendo propostas como formas de tratamento para a IU. No entanto, tais cirurgias, são recursos invasivos que podem ocasionar complicações (infecção, sangramento, lesões ou falha durante a cirurgia), necessitam de tempo de recuperação longo e nem sempre alcançam êxito em seus procedimentos. Já as medicações como anti-inflamatórios, por terem uso contínuo podem resultar em efeitos colaterais indesejáveis (NEUMANN PB et. al., 2005).

Há vários métodos de tratamento conservadores (repouso, medicação, fisioterapia) para o tratamento da incontinência urinária. São recomendados como primeira opção de tratamento por serem menos invasivo e menos perigoso, entre eles destacam-se os Exercícios de Kegel que são feitos para fortalecer os músculos do assoalho pélvico e melhorar a função do esfíncter uretral. Esses exercícios vêm sendo muito estudado pela fisioterapia uroginecológica. Além disso, estudos mostram resultados significativos no que se refere ao tratamento de pacientes que apresentam o quadro de incontinência urinária de esforço (SOUZA JG et al., 2017; TOPUZ S e SEVIG EU, 2016).

REAS/EJCH | Vol. Sup. 26 | e734 | DOI: https://doi.org/10.25248/reas.e734.2019 Página 2 de 8 
Dessa forma, o principal objetivo desse estudo é analisar os efeitos dos Exercícios de Kegel em idosas com incontinência urinária (IU), através de uma revisão sistemática. Assim, com esse trabalho pretendeu-se mostrar a importância da fisioterapia em idosos com incontinência urinaria, após o tratamento com os Exercícios de Kegel e descrever pelos os estudos elegíveis, os benefícios trazidos pelo trabalho do fisioterapeuta na utilização dos exercícios de Kegel para o tratamento de idosas com incontinência urinária.

\section{MÉTODOS}

Uma revisão sistemática é utilizada quando se pretende aplicar estratégias científicas selecione artigos para avaliá-los com um espírito crítico e utilizar todos os estudos relevantes para responder as perguntas feitas no início do estudo (BOTELHO LL, et al., 2011).

Para a obtenção dos resultados foi realizada uma busca em diversos Bases de dados, como Medical Literature Analysis and Retrieval System Online (MedLine), Scientific Electronic Library (SciELO), Literatura Latino-Americana em Ciências da Saúde (LILACS) utilizando os descritores "incontinência urinária", "idoso" e "Exercícios de Kegel" todos disponíveis nos Descritores em Ciência da Saúde (DeCS) e combinando entre as palavras o descritor booleano AND. Para a escolha dos artigos empregou-se os critérios de inclusão, sendo eles: artigos que possui publicação do ano 2010 a 2018; que tenha como participantes da intervenção pessoas com incontinência urinária em idosas; artigos que utilizem como tratamento os exercícios de kegel e que tenham sido publicados em idioma português e inglês. Assim, serão excluídos artigos secundários (revisões bibliográficas, sistemáticas, integrativas, por exemplo); artigos pagos; trabalhos que consta apenas o resumo publicado (incompletos); e aqueles que os exercícios de kegel não foi aplicado por fisioterapeuta. Portanto, a pergunta norteadora está assim delineada: quais os efeitos do exercício de Kegel para o tratamento de idosos com incontinência urinária atendidos por fisioterapeutas?

\section{RESULTADOS}

Os resultados serão apresentados através de uma tabela, mostrando alguns itens importantes (tipo de intervenção e quantidade de sessões, ano de publicação, autores, resultados e população) de todos os artigos. Além disso, foi feito um fluxograma que mostra todas as etapas realizadas junto com as quantidades (n) de artigos incluídos e excluídos, conforme mostra a Figura 1.

Figura 1 - Fluxograma da captação dos artigos que serão selecionados.

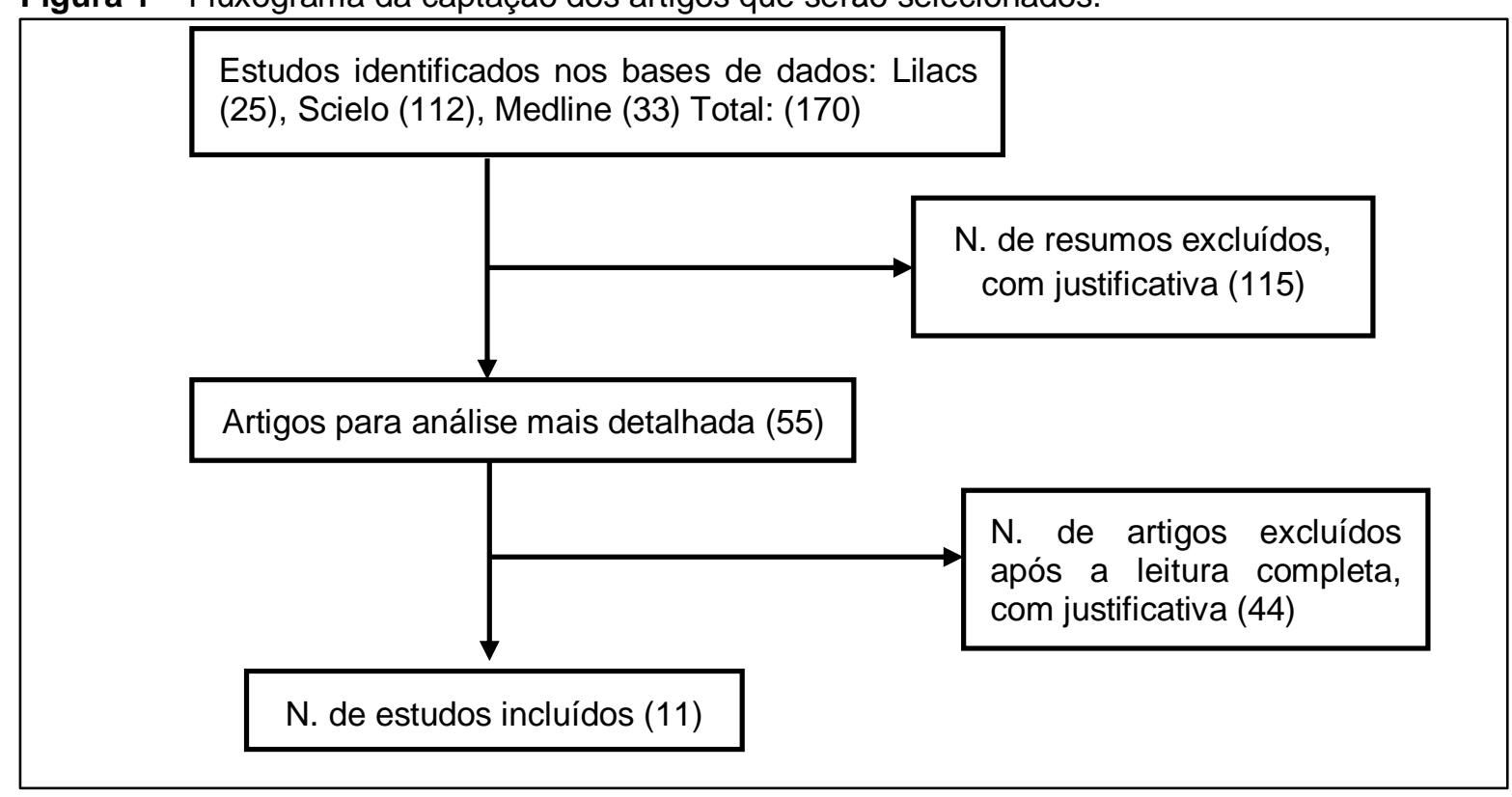

Fonte: Dados da pesquisa, 2019.

REAS/EJCH | Vol. Sup. 26 | e734 | DOI: https://doi.org/10.25248/reas.e734.2019 Página $\mathbf{3}$ de 8 
No caso do Quadro 1 são mostrados os artigos incluídos para esta pesquisa:

Quadro 1 - Caracterização dos artigos incluídos na pesquisa.

\begin{tabular}{|c|c|c|c|}
\hline AUTOR (ANO) & POPULAÇÃO & TIPO DE INTERVENÇÃO E SESSÕES & RESULTADOS \\
\hline $\begin{array}{l}\text { Beuttenmüller } \\
\text { L, et al. (2011) }\end{array}$ & $\begin{array}{l}71 \text { mulheres dividida } \\
\text { em três grupos: } \\
\text { eletroterapia com } \\
\text { exercícios (GEE, } \\
\mathrm{n}=24) \text { exercícios } \\
\text { exclusivamente (GE, } \\
\mathrm{n}=25) \text { e controle (GC, } \\
\mathrm{n}=22) .\end{array}$ & $\begin{array}{l}\text { Eletroterapia e exercícios perineais ( } 12 \\
\text { sessões com duração de } 20 \text { minutos) }\end{array}$ & $\begin{array}{l}\text { Os resultados apontam melhora da contração dos MAP } \\
\text { para os grupos GEE e GE em relação ao GC. Os } \\
\text { exercícios foram eficazes na melhora da contração dos } \\
\text { MAP em mulheres com IUE, sem diferença entre o grupo } \\
\text { de eletroterapia mais exercícios em relação ao grupo de } \\
\text { exercícios. }\end{array}$ \\
\hline $\begin{array}{l}\text { Bruno GS, et al. } \\
(2016)\end{array}$ & $\begin{array}{l}7 \text { mulheres na faixa } \\
\text { etária entre } 35 \text { a } 60 \\
\text { anos }\end{array}$ & $\begin{array}{l}\text { Série de } 10 \text { exercícios de Kegel, com } \\
\text { duração de } 45 \text { minutos, duas vezes por } \\
\text { semana, totalizando } 10 \text { sessões. }\end{array}$ & $\begin{array}{l}\text { O tratamento conservador cinesioterapêutico adotado foi } \\
\text { eficaz no fortalecer a musculatura pélvica para a melhora } \\
\text { da qualidade de vida. }\end{array}$ \\
\hline Eder SE (2014) & $\begin{array}{l}83 \text { pessoas entre } 21 \\
\text { e } 75 \text { anos, sendo a } \\
\text { maioria idosas. }\end{array}$ & $\begin{array}{l}\text { Exercícios EmbaGYN com Exercícios de } \\
\text { Kegel }\end{array}$ & $\begin{array}{l}\text { Na semana } 12, \text { o número médio de episódios de } \\
\text { incontinência / dia (desfecho primário) caiu } 56,2 \% \text { ( } p= \\
0,152) \text {. Um decréscimo de } \geq 50 \% \text { em relação aos valores } \\
\text { basais dos episódios de incontinência foi observado em } \\
65,3 \% \text { dos indivíduos ( } p=0,006) \text {. O número médio de } \\
\text { absorventes por incontinência / dia caiu } 57,1 \%(p=0,001) \text {. } \\
\text { Perda média de urina em consultório de } 24 \text { e } 1 \mathrm{~h} \text { declinou } \\
59,0 \%(p<0,001) \text { e } 67 \%(p=0,019) \text {, respectivamente. }\end{array}$ \\
\hline $\begin{array}{lr}\text { Oliveira } & J R \\
\text { Garcia } & \text { RR } \\
(2011) & \end{array}$ & $\begin{array}{l}11 \text { idosas, média } \\
\text { etária de } 74,2 \text { anos }\end{array}$ & $\begin{array}{l}\text { Cinesioterapia: Exercícios de kegel (10 } \\
\text { sessões) }\end{array}$ & $\begin{array}{l}\text { Considerando o questionário de qualidade de vida, } \\
\text { observou-se redução significativa nas médias e medianas } \\
\text { dos escores em quase todos os domínios. Conclui-se que } \\
\text { a cinesioterapia do assoalho pélvico foi positiva para obter } \\
\text { melhoras sobre a perda de urina diária e alívio dos sinais } \\
\text { e sintomas, bem como na qualidade de vida. }\end{array}$ \\
\hline
\end{tabular}

REAS/EJCH | Vol. Sup. 26 | e734 | DOI: https://doi.org/10.25248/reas.e734.2019 Página 4 de 8 


\begin{tabular}{|c|c|c|c|}
\hline $\begin{array}{l}\text { Porta RO, et al. } \\
\text { (2015) }\end{array}$ & $\begin{array}{l}65 \text { mulheres (35 no } \\
\text { grupo esferas e } 30 \text { no } \\
\text { grupo controle) }\end{array}$ & $\begin{array}{l}\text { Exercícios de kegel com e sem esferas } \\
\text { vaginais (Duração: } 1 \text { mês: duas vezes } \\
\text { ao dia, sendo } 5 \text { dias / semana em casa) }\end{array}$ & $\begin{array}{l}\text { Ambos os tratamentos melhoraram Incontinência urinária, } \\
\text { mas mulheres que realizaram exercícios com esferas } \\
\text { vaginais, apresentaram melhora precoce. Esferas vaginais } \\
\text { foram bem toleradas e seguras. }\end{array}$ \\
\hline $\begin{array}{lr}\text { Ramos } & \text { AL } \mathrm{e} \\
\text { Oliveira } & \text { AAC } \\
\text { (2010) } & \end{array}$ & $\begin{array}{l}08 \text { mulheres com IUE } \\
\text { média de idade } 52,5 \\
\text { anos }\end{array}$ & $\begin{array}{l}\text { Dez sessões de } 40 \text { minutos, três vezes } \\
\text { por semana ( } 21 \text { dias) }\end{array}$ & $\begin{array}{l}\text { A maioria das pacientes passou de perda de urina em jato } \\
\text { para em gotejamento. }\end{array}$ \\
\hline $\begin{array}{l}\text { SHERBURN M, } \\
\text { et al. (2011) }\end{array}$ & $\begin{array}{l}83 \text { Mulheres com IUE } \\
\text { com média de idade } \\
71,6 \text { anos }\end{array}$ & $\begin{array}{l}\mathrm{G} 1-\mathrm{EK}(\mathrm{n}=43) \\
\mathrm{G} 2 \text { - Treinamento Vesical } \\
\text { Durante } 20 \text { semanas. }\end{array}$ & $\begin{array}{l}\text { Ambos os grupos demonstraram melhora no final, mas o } \\
\text { G1 apresentou melhora superior ao G2 quanto à redução } \\
\text { da perda urinária. }\end{array}$ \\
\hline $\begin{array}{l}\text { Silva AM e Oliva } \\
\text { LM (2011) }\end{array}$ & 1 mulher idosa & $\begin{array}{l}10 \text { sessões de fisioterapia com os } \\
\text { exercícios de Kegel associados ao uso } \\
\text { dos cones vaginais. }\end{array}$ & $\begin{array}{l}\text { Concluiu-se que os exercícios de Kegel associados ao uso } \\
\text { dos cones vaginais, nesta paciente, levaram à melhora da } \\
\text { incontinência urinária em um curto período de tratamento. }\end{array}$ \\
\hline $\begin{array}{l}\text { Silva REG, et al. } \\
\text { (2015) }\end{array}$ & $\begin{array}{l}6 \text { participantes } \\
\text { idosas }\end{array}$ & $\begin{array}{l}9 \text { semanas, uma vez por semana } \\
\text { durante } 30 \text { minutos. }\end{array}$ & $\begin{array}{l}\text { Cinco das seis participantes obtiveram consciência da } \\
\text { região vaginal, ou seja, conseguem realizar a contração } \\
\text { ativa da musculatura do assoalho pélvico, podendo realizar } \\
\text { os exercícios sempre que puderem, em casa ou no } \\
\text { trabalho, dando continuidade ao fortalecimento da } \\
\text { musculatura do assoalho pélvico e para a melhora da } \\
\text { qualidade de vida em idosas incontinentes. }\end{array}$ \\
\hline $\begin{array}{l}\text { Souza JG, et al. } \\
\text { (2011) }\end{array}$ & 22 mulheres & $\begin{array}{l}\text { Exercícios de conscientização e da série } \\
\text { de Kegel, realizados duas vezes por } \\
\text { semana, durante } 30 \text { minutos para cada } \\
\text { paciente e totalizando } 12 \text { sessões. }\end{array}$ & $\begin{array}{l}\text { O protocolo cinesioterapêutico adotado foi eficaz para o } \\
\text { fortalecimento da musculatura do assoalho pélvico e para } \\
\text { a } \\
\text { melhora da qualidade de vida em idosas incontinentes. }\end{array}$ \\
\hline $\begin{array}{l}\text { Topuz Ş e Seviğ } \\
\text { EÜ (2016) }\end{array}$ & $\begin{array}{l}30 \text { mulheres para o } \\
\text { grupo de intervenção } \\
\text { e } 30 \text { para o grupo } \\
\text { controle }\end{array}$ & $\begin{array}{l}\text { Exercícios de Kegel } \\
\text { Duração de três meses. Sendo a sessão } \\
\text { a cada duas semanas. }\end{array}$ & $\begin{array}{l}\text { Como resultado do estudo, foi detectado que os exercícios } \\
\text { de kegel tiveram efeito positivo sobre a satisfação sexual } \\
\text { das mulheres. No entanto, a realização de estudos de } \\
\text { amostras de longo prazo e mais abrangentes deve ser } \\
\text { oferecida. }\end{array}$ \\
\hline
\end{tabular}

Fonte: Silveira CM, Cavalcante AA e Ribeiro EGC (2019).

REAS/EJCH | Vol. Sup. 26 | e734 | DOI: https://doi.org/10.25248/reas.e734.2019 Página 5 de 8 


\section{DISCUSSÃO}

\section{Instrumentos utilizados para a avaliação da qualidade de vida}

Dos estudos relatados no Quadro 1 verificou-se que para analisar a qualidade de vida dos pesquisados utilizou-se de três tipos de instrumentos muito conhecidos na fisioterapia. O International Consultation on Incontinence Questionnaire - Short Form (ICIQ-SF) foi utilizado por Porta RO (2015) e Sherburn M et al. (2011), e o Questionário King's Health (KHQ) por Porta RO (2015), Oliveira JR e Garcia RR (2011) e Souza et al. (2011).

O ICIQ-SF com versão em português, validado por Tamanini JTN et al. (2004), é um questionário autoaplicável, simples e breve, que avalia a qualidade de vida de pacientes com IU e sua perda de urina. Composto por quatro perguntas que avaliam a frequência, a gravidade, o impacto da IU e os momentos de perda de Urina. Cada resposta tem uma pontuação e quando somadas mostra o valor do impacto da IU na vida do paciente, podendo ter no máximo 21 pontos. Portanto, quanto maior for a pontuação, mais afetada está a qualidade de vida.

O KHQ avalia o impacto da IU na qualidade de vida composto por trinta questões distribuídas em nove domínios, sendo eles: "percepção da saúde, impacto da incontinência, limitações do desempenho das tarefas, limitação física, limitação social, relacionamento pessoal, emoções, sono, disposição e medidas de gravidade" (SOUZA JG, et al., 2011, p. 42).

Para os autores estes instrumentos foram muito importantes para avaliar a qualidade de vida dos pesquisados. Como por exemplo, no estudo de Souza JG et al. (2011) foi observada uma diminuição dos escores do questionário quando se fez a comparação do momento pré e pós-tratamento, verificando-se melhora na qualidade de vida após o tratamento fisioterapêutico em todos os domínios do questionário.

No caso do trabalho de Oliveira JR e Garcia RR (2011) e Porta RO (2015) utilizou-se o Teste do absorvente (Pad Test) antes de iniciar o tratamento e no fim do mesmo. Também se verificaram resultados positivos e melhoras significativas.

O Pad Test é um método muito simples, não é invasivo e muito bom para avaliar a perda urinária. De acordo com o resultado da perda de urina que é realizado através da pesagem do absorvente íntimo se pode classificar o tipo de IU como leve, moderada ou severa (ABRAMS P, et al., 2002).

Por essa razão, fica evidente que a validação do uso de instrumentos para analisar os sintomas urinários e o grau de incômodo antes e após o tratamento é muito importante, pois permite que haja uma avaliação dos resultados que os Exercícios de Kegel trazem para cada paciente e o seu impacto na qualidade de vida do indivíduo.

\section{Prevalência e causas}

Mesmo que haja prevalência dessa patologia em várias as faixas etárias, dados comprovam que com o aumento da idade (mais de 65 anos) a probabilidade de possuir algum grau de IU é de 8 a $35 \%$. Em geral, cerca de $11 \%$ das mulheres brasileiras sofrem com esse tipo de doença procurando, assim, algum atendimento clínico (OLIVEIRA JR, GARCIA RR, 2011). Estudos mostram que a Incontinência Urinária tem sua causa relacionada com a musculatura flácida do assoalho pélvico. Isso se deve a vários fatores que deixam a mulher mais propícia a ter essa patologia, um deles é: a idade, o fumo, a obesidade, o álcool, etc. (RAMOS AL, OLIVEIRA AAC, 2010).

\section{Efeitos dos Tratamentos para IU}

Sabe-se que a fisioterapia apresenta tratamentos terapêuticos que não oferecem riscos ou incômodos ao paciente. Consiste em treinos específicos que trabalham os músculos afetados na IU normalizando-o e a percepção do corpo pelo paciente (VIANA SBP et al., 2012). Dois artigos encontrados utilizaram em conjunto com os exercícios de kegel outros tipos de tratamento, como Eder SE (2014) que utilizou Exercitador de Assoalho Pélvico EmbaGYN тм, Beuttenmuller L et al. (2011) a eletroterapia e Porta-Roda O (2015) utilizou um grupo com esferas vaginais. O principal resultado do primeiro estudo foi uma diminuição $=50 \%$ da linha 
de base no número episódios de incontinência por dia às 12 semanas ou e o segundo estudo comprovou que tanto os EP isolados quanto associados à eletroterapia para fortalecimento dos MAP podem ser eficazes no tratamento da IU. Na visão de Sousa JG et al. (2011) o tratamento da IU deve ser realizado de forma isolada para cada paciente, mesmo sabendo que possuem os mesmos sintomas e sinais. Por isso, aderir um paciente ao tratamento e obter um sucesso na intervenção é necessário descobrir as consequências que ocasionaram tais fatos. Dessa forma, para melhores resultados Beuttenmuller $L$ et al. (2011) pediu para as pacientes realizarem os exercícios em casa, assim como mudanças de hábitos que pudessem influenciar, positivamente, no controle urinário. Ainda se recomendou a contração do AP nas atividades de esforço.

Vale ressaltar que muito se discutiu nos estudos sobre o desconhecimento dos pacientes sobre a IU. Foi possível perceber que a busca de um tratamento só foi realizada após anos de IU (média de 7 anos). Observou-se também no artigo de Oliveira JR e Garcia RR (2011) o desconhecimento sobre o funcionamento dos músculos do assoalho pélvico. Esse dado foi encontrado em $100 \%$ das participantes do nosso estudo. A falta de interesse em tratamentos, pois muitos acham que faz parte do envelhecimento, e o desconhecimento do assoalho pélvico ocasiona uma demora na procura do atendimento se tornando assim, outro fator de risco: a desinformação.

Beuttenmuller $L$ et al. (2011) afirma que o processo de envelhecimento, à menopausa e o conformismo associado ao desconhecimento de tratamentos existentes afastam essas mulheres na busca de tratamentos efetivos, que, por sua vez, favoreceriam o resgate da vida social, valorizando sua autoestima. É evidente que para obter melhores resultados no fortalecimento dos músculos do assoalho pélvico é necessário que a paciente continue os exercícios ao longo do tempo. Porém, existem várias barreiras existentes como: a falta de informação, a sua disciplina, a disposição para realizá-los, tempo, entre outros. Portanto, o esclarecimento no início do tratamento pelo fisioterapeuta é necessário para que os resultados positivos sejam alcançados com mais relevância.

\section{CONSIDERAÇÕES FINAIS}

Com esse estudo foi possível alcançar os objetivos propostos, pois se concluiu que a fisioterapia tornou eficaz no tratamento da IUE, minimizando as perdas urinárias e melhorando a força do assoalho pélvico. Proporciona, além disso, um maior conhecimento sobre a musculatura desse local, pois nos estudos analisados ficou muito notório que as mulheres não a conheciam e não sabiam realizar a sua contração. $O$ resultado do tratamento depende muito do empenho e comprometimento da equipe e do paciente envolvido. Foi constada nos estudos também a satisfação das pacientes em perceber que houve uma diminuição dos seus sintomas com um tempo pequeno. A mulher com essa doença tem um impacto social grande, pois não pode viajar, dormir fora de casa, passear pela rua, visitar amigos ou familiares, pois sentem medo e ficam envergonhadas caso ocorra micção. Portanto, verifica-se a necessidade de programas que possibilite a difusão e implementação do tratamento da IU pelo fisioterapeuta, para que quando implementados possam oferecer um tratamento convencional menos oneroso, sem custo e com principal foco: a atenção à saúde da mulher, para garantir sua melhor qualidade de vida.

\section{REFERÊNCIAS}

1. ABRAMS $P$, et al. The standardisation of terminology of lower urinary tract function: report from the standardisation sub-committee of the Internacional Continence Society. Neurourology and Urodynamics, 2002; 21(1): 167-178.

2. ALPERIN M, et al. Comparison of pelvic muscle architecture between humans and commonly used laboratory species. Int. Urogynecol. J. 2014; 25: 1507-1515.

3. BEUTTENMULLER $L$, et al. Contração muscular do assoalho pélvico de mulheres com incontinência urinária de esforço submetidas a exercícios e eletroterapia: um estudo randomizado. Fisioterapia e Pesquisa, 2011; 18(3): 2106.

4. BOTELHO LLR, et al. O método da revisão integrativa nos estudos organizacionais. Revista Gestão e Sociedade, $2011 ; 5(11): 121-136$.

5. BRUNO GS, et al. Cinesioterapia aplicada na incontinência urinária feminina de esforço. Revista Eletrônica FACIMEDIT, 2016; 5(1): 18-32.

REAS/EJCH | Vol. Sup. 26 | e734 | DOI: https://doi.org/10.25248/reas.e734.2019 Página 7 de 8 
6. EDER SE. Evaluation of the EmbaGYN ${ }^{\mathrm{TM}}$ pelvic floor muscle stimulator in addition to Kegel exercises for the treatment of female stress urinary incontinence: a prospective, open-label, multicenter, single-arm study. Women's Health, 2014; 10(1): 17-27.

7. NEUMANN PB, GRIMMER KA, GRANT RE, et al. Fisioterapia Para Incontinência Urinária Feminina: um Estudo Multicêntrico Observacional. Journal of Obstetrics and Gynaecology, 2005; 45: 226-232.

8. OLIVEIRA C. Efeitos da Cinesioterapia no Assoalho Pélvico durante o ciclo gravídico-puerperal. Dissertação (Mestrado em Ciências) - Faculdade de Medicina da Universidade de São Paulo, São Paulo, 2006; 106 p.

9. OLIVEIRA JR, GARCIA RR. Cinesioterapia no tratamento da Incontinência Urinária em mulheres idosas. Revista Brasileira de Geriatria e Gerontologia, 2011; 4(2):343-351.

10. PORTA RO, et al. Effect of Vaginal Spheres and Pelvic Floor Muscle Training in Women With Urinary Incontinence: A Randomized, Controlled Trial. Neurourology and Urodynamics, 2015; 34: 533-538.

11. RAMOS AL, OLIVEIRA AAC. Incontinência urinária em mulheres no climatério: efeitos dos Exercícios de Kegel. Revista Hórus, 2010; 4(2): 264-275.

12. REIS LA, TORRES GV. Influência da dor crônica na capacidade funcional de idosos institucionalizados. Revista Brasileira de Enfermagem, 2011; 64(2): 274-80,

13. RODRIGUES MP, et al. Perfil das pacientes do ambulatório de uroginecologia de um Hospital Público de Porto Alegre com relação à incontinência urinária e à qualidade de vida. Revista Clin. Biomed Res. 2016; 36: 135-141.

14. ROIG JJ, et al. Incontinência urinária em idosos institucionalizados no Brasil: uma revisão integrativa. Rev. bras. geriatr. gerontol. 2013; 16(4): 865-879.

15. SHERBURN M, et al. Incontinence improves in older women after intensive pelvic floor muscle training: an assessorblinded randomized controlled trial. Neurourology and Urodynamics, 2011; 30(3): 317-324.

16. SILVA AM, OLIVA LM. Exercícios de Kegel associados ao uso de cones vaginais no tratamento da incontinência urinária: estudo de caso. Revista Scientia Médica. 2011, 21(4): 173-176.

17. SILVA REG, et al. Tratamento cinesioterapêutico como medida de prevenção da incontinência urinária de esforço em mulheres idosas e a sua relação com a qualidade de vida. Journal of Amazon Health Science, 2015; 1(1): 78-81.

18. SOUSA JG, et al. Avaliação da força muscular do assoalho pélvico em idosas com incontinência urinária. Revista Fisioterapia em Movimento, 2011; 24(1): 39-46.

19. TAMANINI JTN, et al. Validação do "King's Health Questionnaire" para o português em mulheres com incontinência urinária. Revista Saúde Pública, 2003; 37(2): 203-11. 